\title{
Comparison of Non-Redundant Array and Double Pinhole Coherence Measurements with Soft X-rays
}

\author{
Gabriel Weil \\ Office of Science, SULI Program \\ Northwestern University \\ Stanford Linear Accelerator Center \\ Menlo Park, California
}

August 25, 2006

\begin{abstract}
Prepared in partial fulfillment of the requirements of the Office of Science, U.S.
Department of Energy Science Undergraduate Laboratory Internship (SULI) Program under the Direction of William Schlotter and Jan Lüning in the Experimental Systems Research Department of the Stanford Synchrotron Radiation Laboratory at the Stanford Linear Accelerator Center.
\end{abstract}

Participant:

Signature

Research Advisor:

Signature

Research Advisor:

Signature 


\section{Table of Contents}

Abstract 3

Introduction 4

Methods and Materials

$\begin{array}{ll}\text { Results } & 8\end{array}$

$\begin{array}{ll}\text { Discussion } & 10\end{array}$

$\begin{array}{ll}\text { Acknowledgements } & 12\end{array}$

$\begin{array}{ll}\text { References } & 12\end{array}$ 


\begin{abstract}
Comparison of Non-Redundant Array and Double Pinhole Coherence Measurements with Soft X-rays, GABRIEL WEIL (Northwestern University, Evanston, IL 60201) WILLIAM SCHLOTTER and JAN LÜNING (Stanford Synchrotron Radiation Laboratory, Menlo Park, CA, 94025)

Experiments on the future Linac Coherent Light Source (LCLS) and other Free Electron Lasers will need to be performed on a single-shot basis. The double pinhole method of measuring spatial coherence requires a separate measurement, with a different pinhole separation distance, for each length scale sampled. This limits its utility for LCLS. A potential alternative uses a Non-Redundant Array (NRA) of apertures designed to probe the coherence over the range of length scales defined by their physical extent, in a single measurement. This approach was tested by comparing diffraction patterns from soft xrays incident on double pinhole and NRA absorption mask structures. The double pinhole fringe visibility data serve as discrete reference points that verify the continuous spectrum of the NRA coherence data. The results present a quantitative analysis of the double pinhole coherence measurements and a qualitative comparison to the NRA images.
\end{abstract}




\section{Introduction}

In order to take advantage of the over ten orders of magnitude increase in peak brilliance anticipated from the LCLS (Linac Coherent Light Source), experiments will need to be designed to operate on a single-shot basis. Such designs must accommodate for shot to shot variations in the source parameters. Since these parameters influence the spatial coherence, it will be important to have a means of measuring spatial coherence on many length scales simultaneously.

The standard method of measuring spatial coherence is to determine the visibility of interference fringes that result from diffraction patterns of radiation incident on double pinhole masks of varying pinhole separations. As seen in Eq. (1), this fringe visibility, V, is directly proportional to the mutual coherence function, $\gamma(\tau)$, with a proportionality constant that depends on the uniformity of the intensities, $\mathrm{I}_{1}$ and $\mathrm{I}_{2}$, incident on the two pinholes [1].

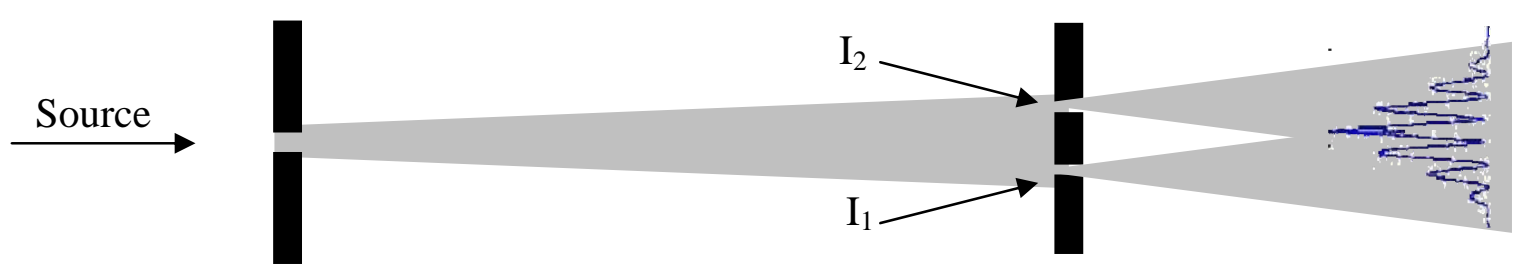

Figure 1: Simplified beam view.

Eq. (1)

$$
V=\frac{2 \sqrt{I_{1}} \sqrt{I_{2}}}{I_{1}+I_{2}}\left|\sim_{12}(\tau)\right|
$$

This method, of course, requires a source whose coherence properties remain stable long enough for several separate measurements. An alternative to this approach uses a Non-Redundant Array (NRA) of apertures arranged to include all possible 
separation distances, thus enabling simultaneous measurements of the spatial coherence of many length scales.

Coherent scattering patterns from the NRA produce images that represent the convolution, see Eq. (2), of the ideal diffraction pattern, $I$, from the set of apertures with the Fourier transform of the mutual coherence function, $C$. Deconvolution can be used to extract the unknown mutual coherence function from the known ideal diffraction pattern [2].

Eq. (2) $\quad(I * C)(x)=\int I(u) C(x+u) d u$

A similar arrangement, known as the Uniformly Redundant Array (URA), was originally developed for use in astronomical imaging [3] and has been applied to soft xray spatial coherence measurements at the Lawrence Livermore National Laboratory laser source [4]. Double pinhole coherence measurement experiments have also been performed on extreme ultraviolet and soft x-ray undulator radiation at the Advanced Light Source [5].

Though today's advanced x-ray sources can be tuned to a certain photon energy, the radiation produced always has finite bandwidth. The energy of greatest photon flux is referred to as the undulator peak, while the point on the higher energy side of the peak where the intensity is about $50 \%$ of its value at the peak is referred to as the blue edge. The blue edge is typically used in coherence experiments because the photons at that energy have taken the straightest path and thus exhibit the lowest degree of divergence. In this experiment, discrete data points from double-pinhole experiments were used to verify the accuracy of the continuous coherence function provided by the NRA analysis. 


\section{Methods and Materials}

The coherence properties of soft x-ray synchrotron radiation were studied by observing the visibility of interference fringes produced by diffraction at the apertures of double pinhole and NRA masks. Absorption films were prepared by depositing a $900 \mathrm{~nm}$

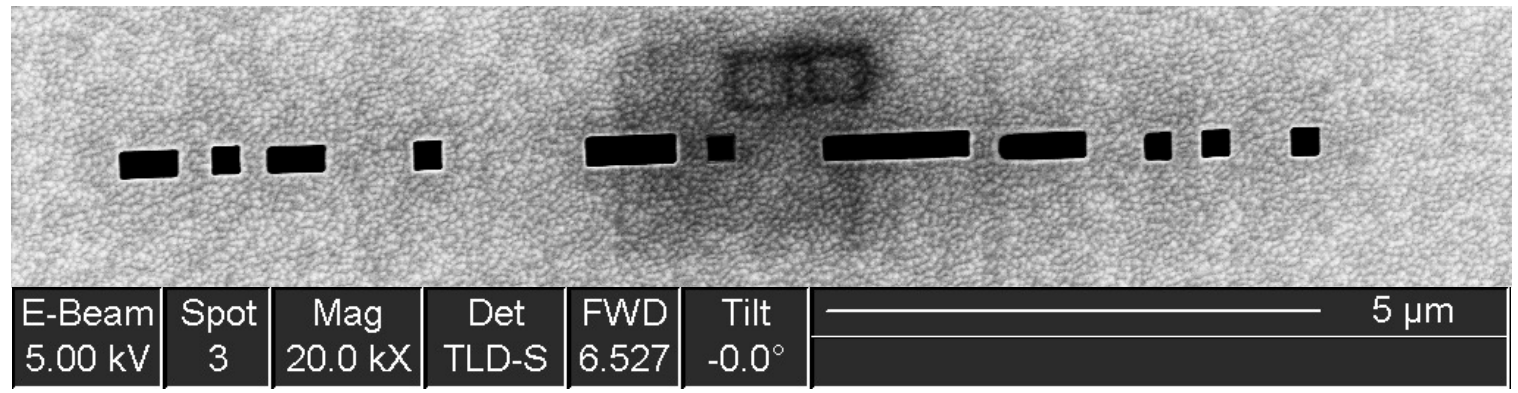

Figure 2: Scanning Electron Microscope (SEM) image of NRA Au mask with aperture separation distances from 0.5 to $12 \mu \mathrm{m}$ and $900 \mathrm{~nm}$ thickness

thick layer of Au onto a $100 \mathrm{~nm}$ thick $\mathrm{Si}_{3} \mathrm{~N}_{4}$ membrane at a rate of $0.6 \mathrm{~nm} / \mathrm{s}$. In order to achieve the requisite aspect ratio, a Focused Ion Beam (FIB) was used to mill double pinhole masks, see Figs. 3 and 4, with center to center distances of 1, 3, 5 and $7 \mu \mathrm{m}$ and pinhole diameters ranging from 100 to $300 \mathrm{~nm}$. The NRA absorption masks were also fabricated using a FIB, see Fig 2.

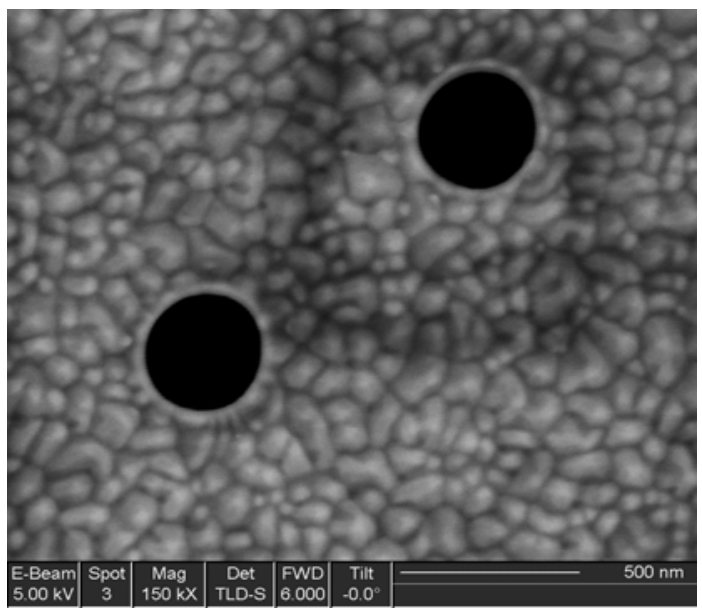

Figure 3: SEM image of double pinhole mask with $1 \mu \mathrm{m}$ separation

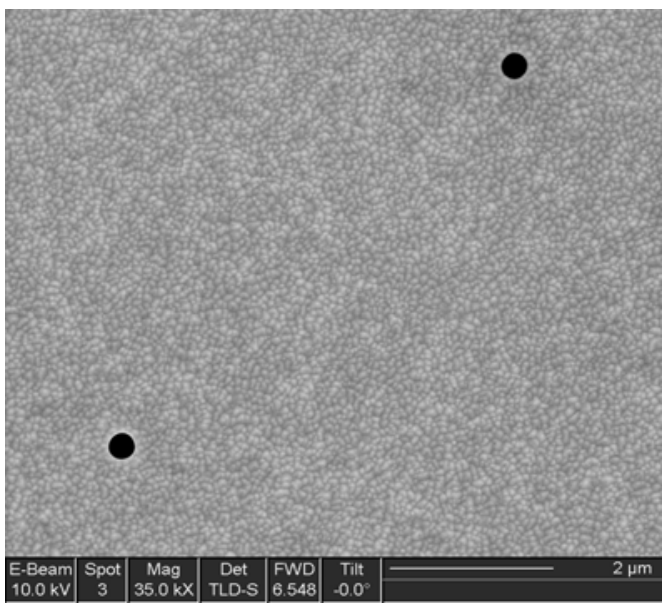

Figure 4: SEM image of double pinhole mask with $7 \mu \mathrm{m}$ separation

To set the groundwork for similar studies on LCLS, the experiment was

performed on the undulator at beamline 5-2 at SSRL, at $420 \mathrm{eV}$. 
As in Fig. 5, the divergence of the beam was controlled by manipulating a set of movable horizontal slits. Images were acquired at four horizontal aperture settings identified by their relative intensity incident on the sample, at $100 \%, 12.7 \%, 5 \%$ and $2.4 \%$ relative intensity. The intensity loss results from constraining the aperture so that the effective source size was reduced, which has the effect of increasing spatial coherence. In all cases, the beam was then focused with a series of spherical mirrors and the energy was selected with a spherical grating monochromator. The resulting radiation was coherently scattered by the double pinhole and NRA samples to form a diffraction pattern on the detector. The CCD camera uses a backside illumination chip for maximum quantum efficiency [6] and uses thermoelectric cooling in order to minimize dark noise The CCD is composed of a $1340 \times 1300$ array of $20 \mu \mathrm{m}$ pitch pixels and the distance between the sample and the detector is $30 \mathrm{~cm}$.

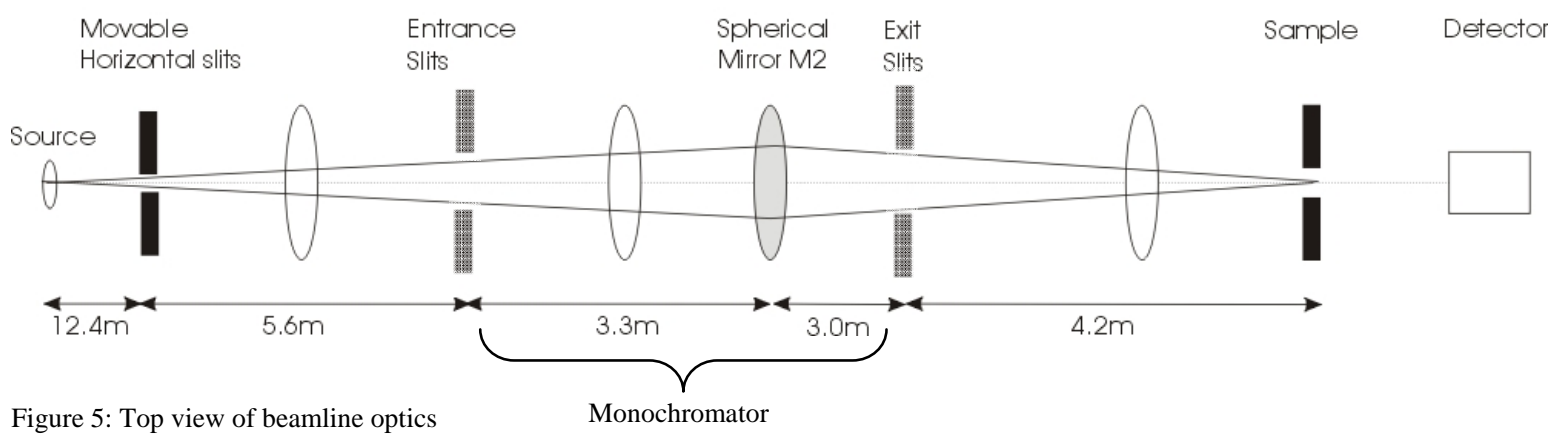

All the aforementioned measurements were made on the blue edge. An additional data set, however, was acquired on the undulator peak for the $12.7 \%$ relative intensity setting.

Double pinhole fringe visibilities were determined by taking horizontal line cuts from 2-dimensional images and sampling maximum-minimum pairs from each line cut to apply the visibility formula: 
Eq. 3

$$
V=\frac{I_{\max }-I_{\min }}{I_{\max }+I_{\min }}
$$

where $I_{\max }$ and $I_{\min }$ are corresponding relative maxima and minima on the intensity curve, as shown in Fig. 6.

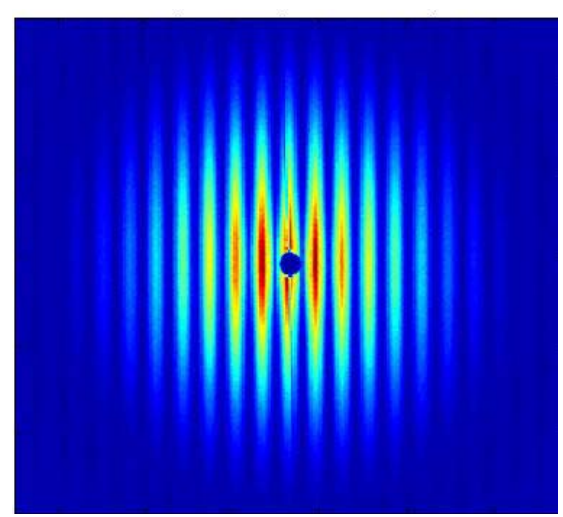

Figure 6:Colorized image from CCD for double pinhole with $3 \mu \mathrm{m}$ separation distance at $5.0 \%$ Relative Intensity

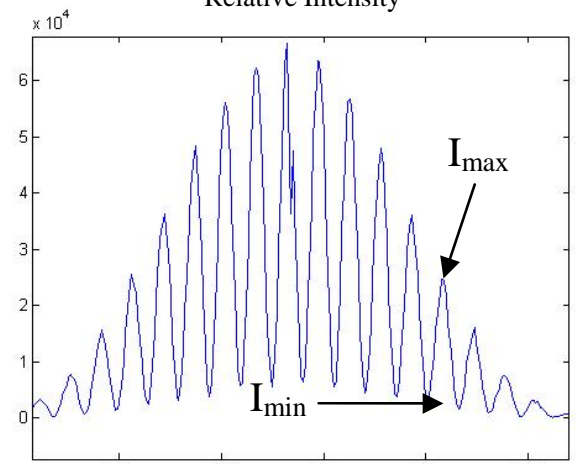

Figure 8: Horizontal line cut plot of intensity vs. position from 6 .

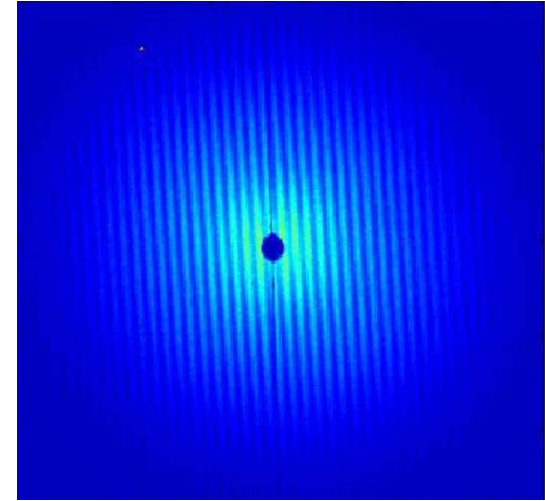

Figure 7: Colorized image from CCD for double pinhole with $7 \mu \mathrm{m}$ separation distance at $12.7 \%$ Relative Intensity

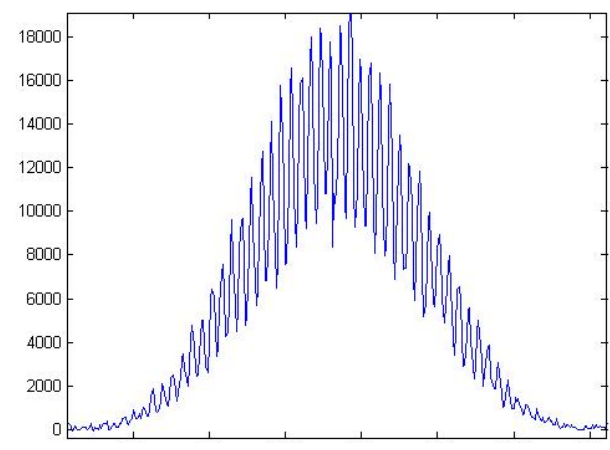

Figure 9: Horizontal line cut plot of intensitv vs. nosition from 7.

Results

The double pinhole data suggest that there is an upper limit on the spatial coherence of the undulator at beamline 5-2 at SSRL at certain length scales. At $2.9 \mu \mathrm{m}$, there is no significant variation between the $12.7 \%, 5.0 \%$ and $2.4 \%$ relative intensity, suggesting that the aperture setting only begins to influence coherence after a certain threshold value that depends on the length scale. This view is supported by the fact the $12.7 \%$ relative intensity curve begins to deviate from the two smaller aperture data sets by the third point at $4.9 \mu \mathrm{m}$ and pulls away completely by the fourth point at $6.8 \mu \mathrm{m}$. The 
$100 \%$ relative intensity curve appears to reach this threshold by the first data point at 1.0 $\mu \mathrm{m}$, as it already shows significantly lower visibility there and the deviation increases steadily with increasing length scale. We might therefore expect the $2.4 \%$ and $5.0 \%$ relative intensity curves to diverge on length scales greater than those investigated in this experiment.

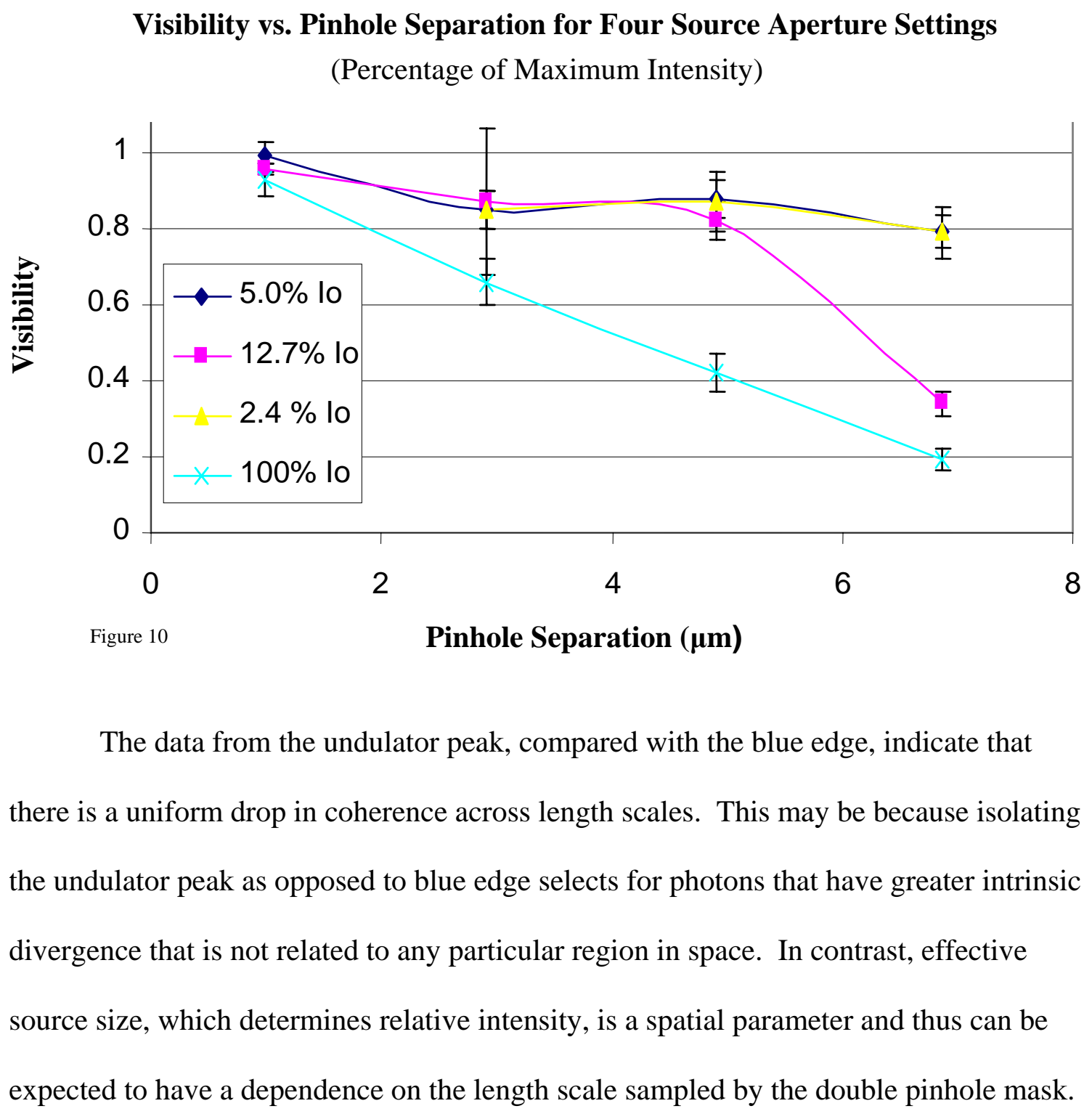




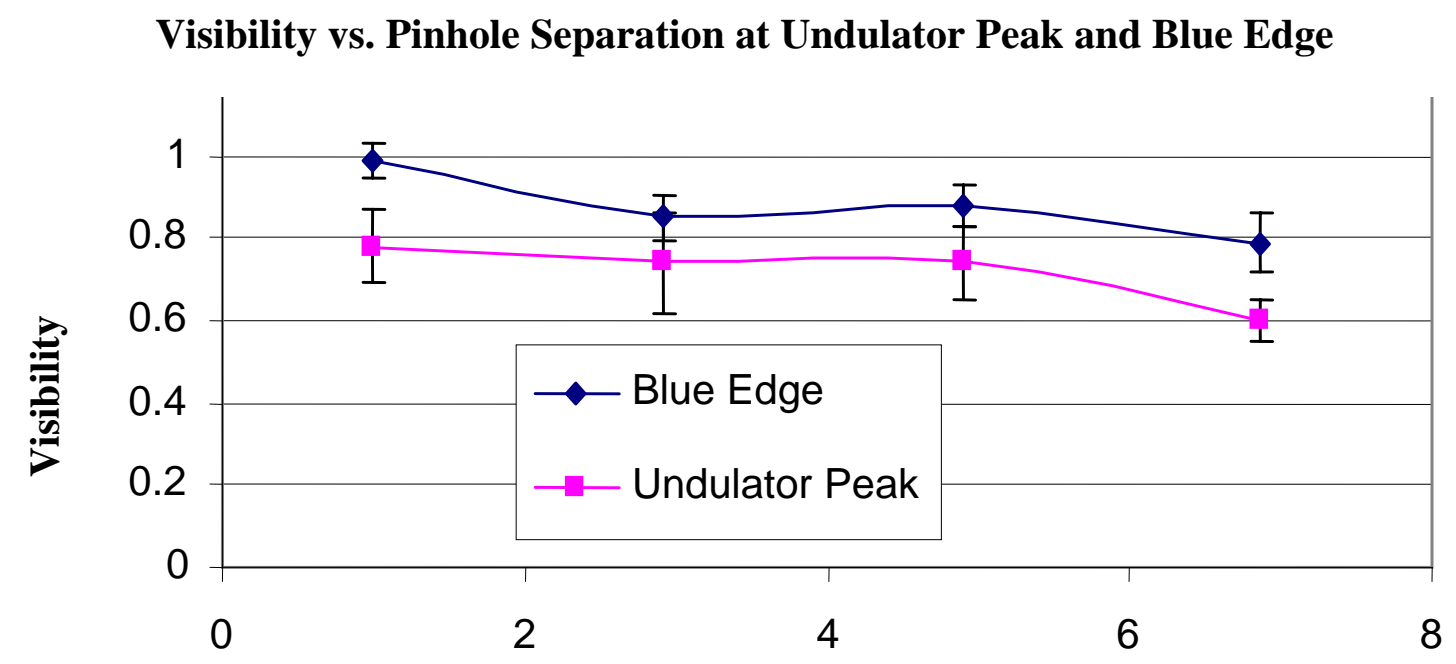

Figure 11

Pinhole Separation $(\mu \mathrm{m})$

The NRA data indicate a

general trend that coherence decreases

with increasing relative intensity.

Since the quantitative analysis is

ongoing, we cannot draw any

conclusion regarding whether the

threshold effects that appear with

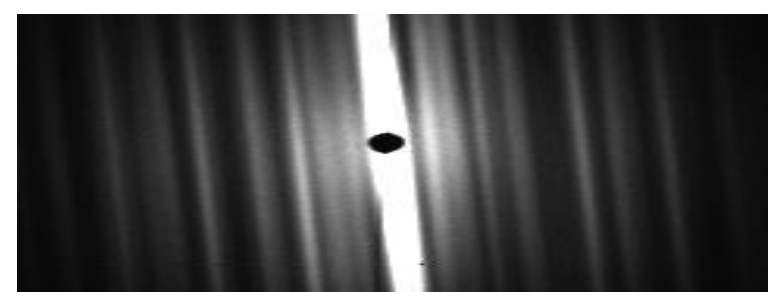

Figure 12: Raw NRA Image at 100\% Relative Intensity

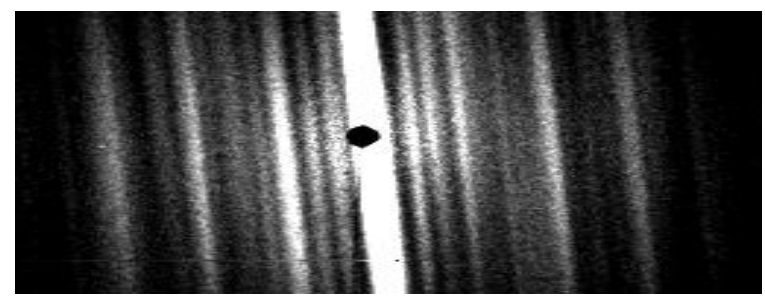

Figure 13: Raw NRA Image at $12.7 \%$ Relative Intensity

separation distance and relative

intensity are detectable in the NRA

images. Analysis of this data is

ongoing.

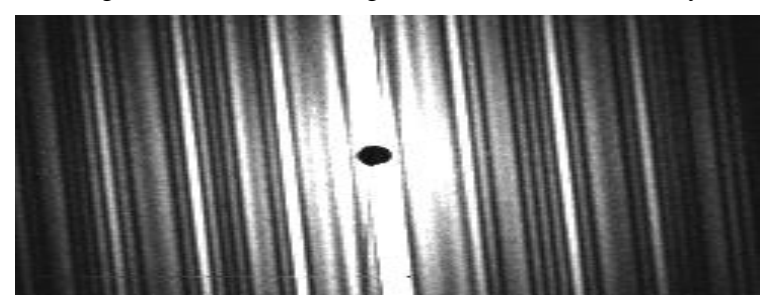

Figure 14: Raw NRA Image at 5.0\% Relative Intensity

\section{Discussion}

The limited spatial resolution of the CCD had the effect of producing systematically lowered visibility measurements, because minima values are raised and maxima values are lowered by offset extrema. This error is more pronounced for larger 
pinhole separation distances where the fringes are more tightly spaced. The statistical error was at acceptable levels and can largely be accounted for by the necessity of maxima and minima coming from different points on the envelope. To cancel out these effects, the intervals were always defined such that the maximum was to the left of the minimum and an equal number of intervals were taken from each side of the central maximum. There was no significant variation in visibility between line cuts for a given image.

The double pinhole data indicate that it will probably not be feasible to produce a control NRA image that represents the ideal diffraction pattern, due to the apparent coherence limit on certain length scales. However, this ideal type is theoretically derivable and should prove useful with higher quality images at various coherence settings.

In order to explore the full range of the NRA and investigate the threshold behavior relating length scale and source size, it would be interesting to use pinholes of greater separation distances. This, however, would exacerbate the resolution problems faced in this experiment and would likely require longer wavelength radiation or increasing the distance between the sample and the CCD to acquire reliable data. Moving to lower photon energy would increase the risk of encountering higher harmonics that would distort the data and moving the detector back would require restructuring of the experimental station, so modifications are required to fully explore this phenomenon.

The NRA method holds promise for application on LCLS and high peak brilliance sources, but further testing is required. The trends visible in the NRA images are encouraging and merit exploration. Advances in this area will allow more effective use of LCLS and other Free Electron Lasers to study coherence on ultra-fast time scales. 


\section{Acknowledgements}

The author would like to thank U.S. Department of Energy, Office of Science for providing me with the opportunity to participate in the SULI program. I would also like to thank my mentors, William Schlotter and Jan Lüning, as well as Ramon Rick and all the other members of the group that supported me. Portions of this research were carried out at the Stanford Synchrotron Radiation Laboratory, a national user facility operated by Stanford University on behalf of the United States Department of Energy, Office of Basic Energy Science.

\section{References}

[1] Eugene Hecht Optics, $3^{\text {rd }}$ edition, Addison Wesley Longman: Menlo Park, California, 1998.

[2] Private consultation with Andreas Scherz, postdoctoral fellow at Stanford Synchrotron Radiation Laboratory.

[3] O. I. Vassilieva, R. C. Chaney "Method for reducing background artifacts from images in single-photon emission computed tomography with a uniformly redundant array coded aperture,” Applied Optics, vol. 41, no. 70, pp. 1454- 1461, March 2002.

[4] K. A. Nugent, J. E. Trebes "Coherence Measurement for short-wavelength light sources,” Rev. Sci. Instrum. Vol. 63, no. 4, pp. 2146-2151, April 1992.

[5] K. M. Rosfjord, Y. Liu, D. T. Attwood, “Tunable coherent soft x-ray,” IEEE Journal of Selected Topics Quantum Electronics, Vol. 10 no. 6, November 2004.

[6] Princeton Instruments / Acton, PI-MTE, Fact Sheet, http://www.piacton.com 